\title{
Impact Characteristic Analysis of Hydraulically Actuated Quadruped Robots During Running
}

\author{
GE Yuan Yuan ${ }^{1, a}$, Zhang Hong Ji ${ }^{1, b}$ \\ ${ }^{1}$ Research Center of Contemporary Design and Advanced Manufacturing Technology, Yulin \\ University, Shaanxi Yulin 719000, China
}

aGe_yuanyuan022@163.com, bHongji_258@163.com

\begin{abstract}
Keywords: Run moving; Touchdown impact; Hydraulic drive; Quadruped robots; Dynamic model Abstract. When the quadruped robot run high-speed movement, Frequent or large impact force from the ground on the robot system cause significant damage to stability and reliability of the hydraulic system and robot itself. Through decomposition the high-speed running process, the touchdown, slippage, and stationary state dynamic model of robot mechanism are established. By analyzing the running state, the influence of the ground impact force on the structure of the body and the characteristic curve of the hip joint and knee joint movement are obtained. Through the establishment of impact dynamics equation, using the Matlab and Adams dynamic simulation software, carried out simulation experimental research on the impact force on the ground when the robot running. The results can provide a reliable basis and theory of control for the design of zero impact gait planning and high speed gait planning.
\end{abstract}

\section{Introduction}

The mobile technology of the multi-legged robot is actually a bionic technology. The gait and posture parameters of the multi-legged robot are obtained directly from the research of walking posture of the animal [1]. Animal walking is a coordinated action by the flexibility of the bones, muscles and joints of the legs [2]. The multilegged walking robot's mechanical system mainly refers to the layout of the leg mechanism, form and quantity, etc., which is closely associated with the overall structure, and mechanical structure characteristics of robot legs, directly determine the kinematics and dynamics characteristics of the robot [3]. So leg mechanism is an important part of multi-legged walking robot, which is one of the keys of mechanical design. The four-legged robot has better stability and strong carrying capacity than the bipedal robot, and it is simpler and easier to control than the six-legged and eight-legged walking robot [4]. Therefore, four-legged robot has more obvious advantages than other foot robots. At present, high load and high speed performance are an important index of the foot robot, and foreign scholars have done a lot of research on it. Famous research results at home and abroad include BigDog, AlphaDog, LittleDog and Cheetah developed by Boston dynamics [5]. HyQ robot developed by IIT university of Italy [6], Hyperion 4 robot developed by Japan [7], Hydraulic driven quadruped bionic robot developed by shandong university [8]. In the research of four-legged walking robot, the key to improve walking speed is to use dynamic walking instead of static walking. However, as the speed of walking increases, a serious problem arises-from the impact on the robot body. Due to the influence of ground recovery coefficient and friction coefficient, frequent or large impact vibration will cause the robot to slip and bounce twice, thus damaging the direct transition between typical gaits. Due to the abrupt change of the robot's motion state after the impact, it may also lead to unstable walking or damage to the body [9]. How to reduce the impact has become an urgent problem to be solved in the development of walking robot technology.

In this paper, a new type of four-legged hydraulic buffer leg mechanism is studied. Through the process of robot jumping movement decomposition, respectively establish robot touchdown, slippage and stationary state dynamic dynamics and the running state of the movement gait analysis, simulation and inverse solution. The dynamic model of leg mechanism is used to simulate the joint 
trajectory tracking control and impact force simulation. It provides theoretical basis for solving the impact of high speed and heavy load.

\section{The establishment of dynamics model}

During the robot running, the contact process between the toe and the ground can be divided into three stages: impact contact, slippage and static. The dynamic model of three stages was established respectively. The robot enters the ground impact stage immediately after starting to walk. At the point of the toe touches the ground, when $t=t_{\mathrm{c}}$. Due to the sudden change in the speed of the toes when touching the ground, there will be an impact on the toes and the ground. Because the impact time is very short, the constraint reaction against of the toe is considered as an instantaneous impact [10]. In order to accurately simulate the change of the motion process and the impact force, a generalized binding force $T_{\varepsilon}$ is introduced. The impact contact dynamic model can be established when touchdown.

$$
D^{\delta}(q)+C^{\delta}(q, \phi)\left(\alpha+G^{\delta}(q)=T^{\delta}+\Gamma^{\delta}\right.
$$

$\left[D^{\delta}(q)\right]^{-1}$ is the inverse of the inertial matrix for the robot system. Because the inertial matrix $D^{\delta}(q)$ is a symmetric positive definite matrix and is nonsingular. So both matrix $\left[D^{\delta}(q)\right]^{-1}$ and $J\left[D^{\delta}(q)\right]^{-1} J^{T}$ are symmetric positive definite matrices, and they're both invertible.

\section{Sliding dynamics model}

The robot has experienced the touchdown stage after the movement in the process, due to the impact time is short and the motion state remains the same. Therefore, in the high speed movement the toe and the ground will produce relative horizontal sliding motion [11]. The force exerted by the momentum transfer between the toe and the ground is the main force in the process. According to the overall force analysis of the robot system, the sliding dynamics model of the system is as follows

$$
\left[M E M_{r}^{-T}\right]\left[\begin{array}{c}
v_{0} \\
\omega_{0}
\end{array}\right]+J_{T W} \otimes=\left[\begin{array}{c}
f_{x}+\sum_{i=1}^{n} f_{\mathrm{a} i} \\
f_{y}+\sum_{i=1}^{n} N_{i} \\
f_{z}+\sum_{i=1}^{n} f_{n i}
\end{array}\right]
$$

Where, $M$ is the mass of the whole system. $f_{a i}$ and $f_{n i}$ are the longitudinal and lateral forces of the toe. $\mu$ is the ground sliding friction coefficient. $\Theta$ is the rotational angular velocity of each joint angle of the robot. $f$ is the force of friction on the toe.

$$
\begin{gathered}
J_{T W}=\sum_{0}^{i} m_{i} J_{\pi i} \\
J_{\pi_{i}}=\left[k_{1} \times\left(r_{i}-p_{1}\right), k_{2} \times\left(r_{i}-p_{2}\right), \mathrm{L}, k_{i} \times\left(r_{i}-p_{i}\right)\right]
\end{gathered}
$$

\section{Static dynamics model}

The dynamic modeling of static stage is mainly to solve the horizontal force of the robot. The vertical inertial force at the toe is $F_{y}$, the horizontal inertial force $F_{x}$. According to Newton's law, the kinetic equation of the static moment is

$$
\begin{aligned}
& F_{x}=\sum_{i=1}^{n} m_{i} a_{x}+F_{f} \\
& F_{y}=\sum_{i=1}^{n} m_{i}\left(a_{y}+g\right)
\end{aligned}
$$

Where, $a_{x}$ and $a_{y}$ respectively represent the acceleration level and vertical component of the body, $F_{f}$ indicates the force of friction on the foot. 


\section{Impact analysis}

In the process of robot running, the following assumptions are made for the impact of the system. The impact is completed in a very short period of time. The toe and the ground are the point contact. The dynamic theory indicates that the system is in the impact process the Laplace equation is [12].

$$
\frac{\partial}{\partial t}\left(\frac{\partial T}{\partial \&}\right)-\frac{\partial T}{\partial q}+\frac{\partial P_{g}}{\partial q}+\frac{\partial P_{k}}{\partial q}+\frac{\partial P_{I}}{\partial q}=\tau_{i}
$$

$P_{I}$ is the impulse potential energy of the system. $\tau_{i}$ is the torque applied to joint $i$. In the short time within $\Delta t$ of the impact process, the derivative of the equation (11) with respect to $t$. And order $\Delta t--0$, there is

$$
d\left[\frac{d}{d t}\left(\frac{\partial T}{\partial \&}\right)\right]+\frac{\partial \mathcal{R}_{I}}{\partial q}=0
$$

Suppose the touchdown leg of a robot is affected by the impulse $I$ in $\Delta t$ time. The direction is $N=\left[0, N_{x}, N_{y}, 0\right]^{T}$ in absolute coordinates. The impulse potential of its relative to absolute coordinate system is

$$
R_{I}^{\alpha}=-N \cdot I \cdot H \cdot L
$$

Where, $H$ is the $4 \times 4$ coordinate transformation matrix and $L$ is the collision contact point coordinate. The dynamic equations of the robot can be obtained as follow.

$$
M(q) \cdot d \notin=N \cdot I \cdot \frac{\partial H}{\partial q_{i}} \cdot l
$$

In the formula, $d \oint$ is the change of velocity before and after the collision.

$\Delta t$ can be derived from the following formula

$$
I=F \cdot \Delta t
$$

$$
\Delta t=\frac{2(1+e) \cdot h_{1}}{\sqrt{2 g h_{2}}}
$$

In the formula, $h_{1}$ and $h_{2}$ are the distance between the center of mass and the center of the robot when it falls on the ground. $e$ is the recovery coefficient in completely inelastic collision.

\section{Simulated analysis}

A complete hydraulic four-legged robot is generally composed of three parts: body, leg mechanism and hydraulic system. The model parameters are shown in table 1

Table1 The model parameters of quadruped robot

\begin{tabular}{cccc}
\hline System element & Quality $/ \mathrm{kg}$ & Moment of inertia $/ \mathrm{kg} . \mathrm{mm}^{2}$ & Length $/ \mathrm{mm}$ \\
\hline Engine body & 58.75 & $8.25 \mathrm{e}+006$ & 800 \\
Thigh & 3.25 & 654.17 & 360 \\
Shank & 2.64 & 566.8 & 280 \\
Hydraulic cylinder of shank & 3.65 & 473.48 & 340 \\
Drive hydraulic cylinder & 0.16 & 7.67 & 200 \\
\hline
\end{tabular}

In this paper, design of hydraulic drive quadruped robot structure diagram as shown in figure 1 , the hip and knee can be achieved respectively $120^{\circ}$ range of movement, the leg length of hydraulic cylinder and drives the hydraulic cylinder telescopic divided into for $80 \mathrm{~mm}$ and $60 \mathrm{~mm}$.

According to the phase transition of running motion the four-legged is known, the four legged robot can be decomposed into four stages, namely the hind legs power, the body rise high into the air, the front legs touched the ground and the joint contraction. According to the parameters and kinematic parameters of the robot, using the Matlab software, impact process of quadruped robot touches the ground has been simulated. 


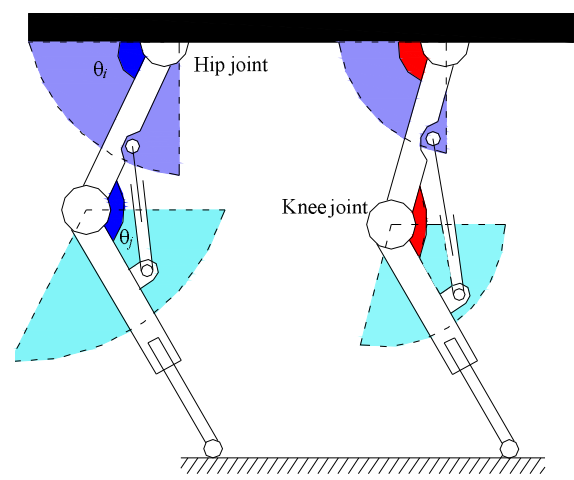

Fig.1 Diagram of quadruped robot model structure

The body centroid trajectory as shown in figure 3 during a full running movement. According to the motion law of the center of mass, it can be seen that, the four-legged robot is slowly rising after the center of mass decline in the peak of the toe in the process of running. This is due to the rapid decline of the body due to the robot's gravity, and then the body starts to rise again due to the effect of the hydraulic buffer. The gait of the robot during the full running cycle is shown in figure 2 .

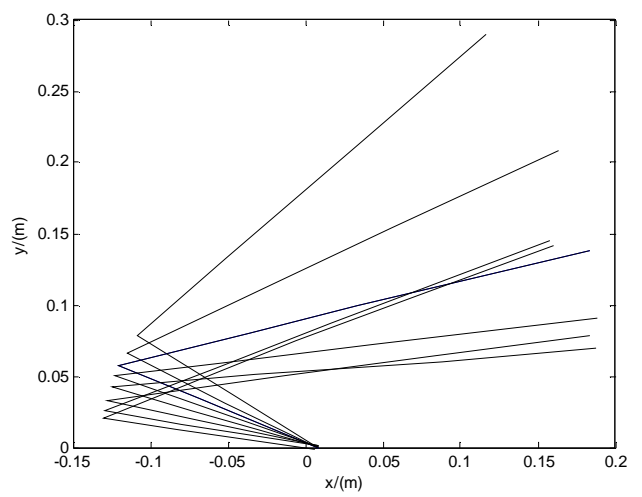

Fig.2 The gait of the robot landing stage

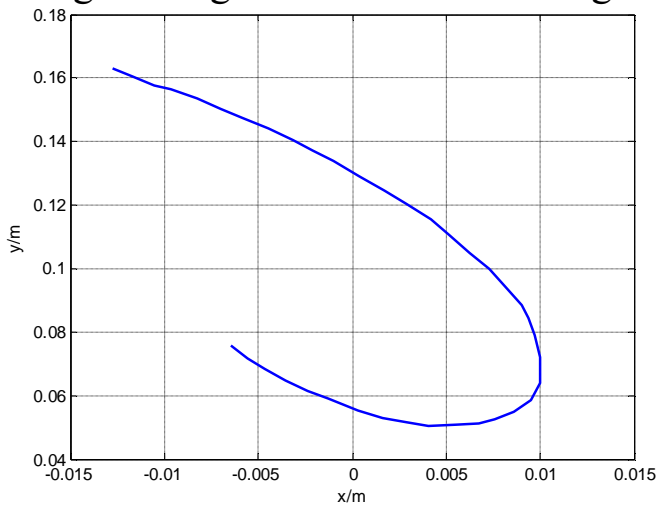

Fig.3 The movement of the center of mass during the landing phase of the quadruped robot

The simulation of running motion of the four-legged robot designed in this paper is set up, and the simulation process is set for the robot to jump at the initial speed of $0.2 \mathrm{~m} / \mathrm{s}$. From the first jump to the next jump, the time to complete a run is $5 \mathrm{~s}$. Fig. 4 shows the curve of acceleration in the running process. It can be seen from Fig. 4 that the peak acceleration value of the front and rear ends of a complete running movement is basically constant, and the acceleration value at the rear point is greater than the acceleration value of the front toe. This is because the forelegs are in a state of the ground, before the robot by controlling the robot hind legs completely rise high into the air, the acceleration value of brief keep calf hydraulic cylinder and the knee joint, to withstand the impact from the ground, get larger upward acceleration at the same time, prepare the next jump. To determine the size of the impact force from the ground during running. As shown in Fig. 5, can be seen from Fig. 5 , the forelegs are the first to hit the ground during the impact, and then the hind legs. Since the 
forelegs first touched the ground, the time and impact of contact with the ground were relatively large. Compared to the relationship between the impact and time of the forelegs of the robot, the two hind legs are simultaneously landing, and the size of the impact force is less than the front leg. As the robot is running, the forelegs is the main force part, while the hind leg is the main power part, so the hind leg is less impact than the forelegs.
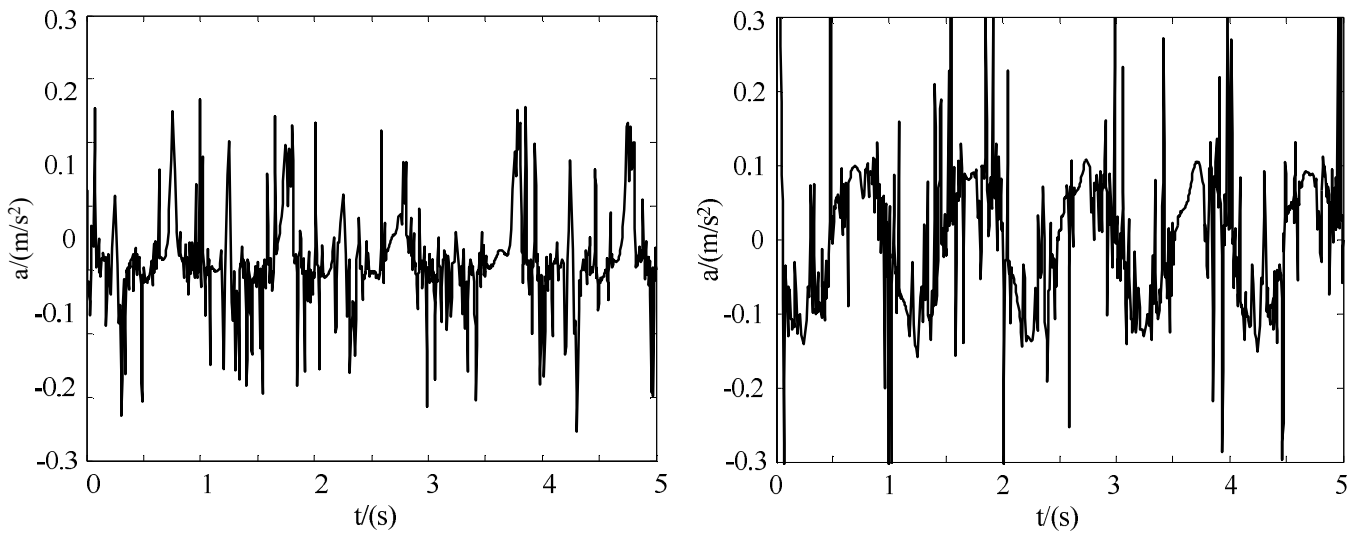

(a) The acceleration curve of forelegs

(b) The acceleration curve of hind legs

Fig.4 The acceleration variation curve of the robot's running

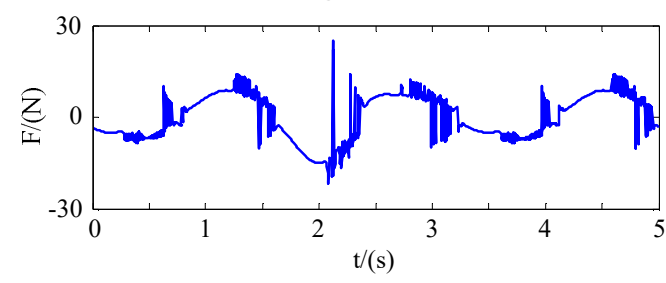

Fore leg

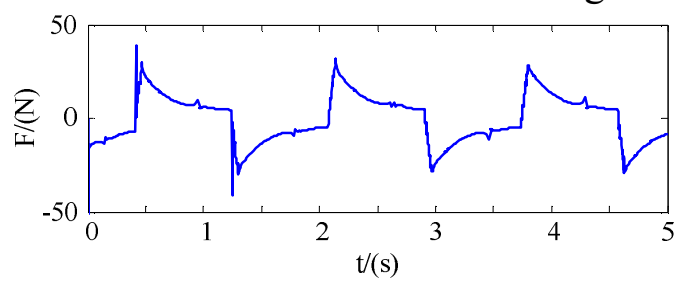

Hind leg

Fig.5 The impact of the toe when the robot landing

\section{Conclusions}

This paper presents a numerical analysis method for the impact force of a four-legged robot running at high speed. The dynamics and models of three stages (contact, slippage and static) of the four-legged robot are established. The state analysis of high speed running is carried out. The motion simulation of the four-legged robot's center of mass with the speed of $0.2 \mathrm{~m} / \mathrm{s}$ and the impact force on the ground was achieved. The simulation results show that the impact of the ground during the high-speed movement of the four-legged robot has great influence on the structural stability of the body and the hydraulic system itself. The joint angle curve is smooth in the landing stage of the robot, there is no mutation in the angular velocity and torque curve of the joint, and the change trend is relieved, indicating that the robot has a stable overall performance during the movement. The hind legs suffer less impact when they hit the ground than the forelegs. It provides a reliable theoretical basis for the gait planning and zero impact gait planning for the following four-legged robot high-speed motion.

\section{Acknowledgements}

This work was financially supported by the Science and Technology of Shaanxi Province (No. 17GK0895).

\section{References}

[1] Tian Xinhua, Gao Feng and Chen Xianbao: J. Mech. Eng. Vol. 49 (2013), p. 81-88.

[2] Li Yibin, Li Bin and Rong Xuewen: J. Shandong University (Engineering Science), Vol. 41 (2011), p. 32-36. 
[3] Liu Q, Chen X and Han B: J. Bionic Eng. Vol. 14( 2017), p. 218-231.

[4] Na B, Choi H and Kong K: Mechatronics IEEE/ASME Transactions, Vol. 20 (2015), p. 924-933.

[5] Raibert M, Blankspoor K and Nelson G. Big-dog the rough-terrain quadruped robot. Proceedings of 17th World Congress, The International Federation of Automatic Control, Seou, Korea, 2008: 6-9.

[6] Kalakrishnan M, Buchli J and Pastor P: Inter. J. Robotics Research, Vol. 30 (2011), p. 236-258.

[7] Semini C, Tsafarakis N G and Guglielmino E: Proc. IMechE Part I: J. Systems \& Control Eng. Vol. 225 (2011), p. 831-849.

[8] Wei Baochen, Gao Feng: Frontiers of Mechanical Eng. Vol.7 (2012), p. 109-119.

[9] Zhang Xuefeng, Qin Xiansheng and Feng Huashan: Robot, Vol.35 (2013), p. 582-588.

[10] Zhang Xuefeng, Qin Xiansheng and Feng Huashan: Robot, Vol. 35 (2013), p. 135-141

[11] Dong J H, Sang O S and Lee J: Inter. J.Robotics Research, Vol. 33 (2014), p. 1417-1445.

[12] Spröwitz A., Tuleu A. and Vespignani M.: Inter. J. Robotics Research, Vol. 32 (2013), p. 932-950. 